Journal of

Applied

Crystallography

ISSN 0021-8898

Editor: Anke R. Pyzalla

\title{
Characterization of order domains in $\gamma$-TiAl by orientation microscopy based on electron backscatter diffraction
}

\author{
Claudio Zambaldi, Stefan Zaefferer and Stuart I. Wright
}

J. Appl. Cryst. (2009). 42, 1092-1101

Copyright (C) International Union of Crystallography

Author(s) of this paper may load this reprint on their own web site or institutional repository provided that this cover page is retained. Republication of this article or its storage in electronic databases other than as specified above is not permitted without prior permission in writing from the IUCr.

For further information see http://journals.iucr.org/services/authorrights.html

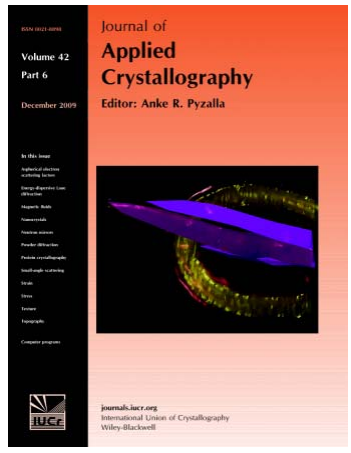

Many research topics in condensed matter research, materials science and the life sciences make use of crystallographic methods to study crystalline and non-crystalline matter with neutrons, X-rays and electrons. Articles published in the Journal of Applied Crystallography focus on these methods and their use in identifying structural and diffusioncontrolled phase transformations, structure-property relationships, structural changes of defects, interfaces and surfaces, etc. Developments of instrumentation and crystallographic apparatus, theory and interpretation, numerical analysis and other related subjects are also covered. The journal is the primary place where crystallographic computer program information is published.

Crystallography Journals Online is available from journals.iucr.org 
Journal of

Applied

Crystallography

ISSN 0021-8898

Received 20 February 2009

Accepted 9 September 2009

(C) 2009 International Union of Crystallography Printed in Singapore - all rights reserved

\section{Characterization of order domains in $\gamma$-TiAl by orientation microscopy based on electron backscatter diffraction}

\author{
Claudio Zambaldi, ${ }^{a}$ Stefan Zaefferer ${ }^{\mathrm{a} *}$ and Stuart I. Wright ${ }^{\mathrm{b}}$ \\ ${ }^{a}$ Microstructure Physics and Metal Forming, Max-Planck-Institute für Eisenforschung, Düsseldorf, \\ Germany, and ${ }^{\mathbf{b}} \mathrm{EDAX} / \mathrm{TSL}$, Draper, UT, USA. Correspondence e-mail: s.zaefferer@mpie.de
}

\begin{abstract}
A new approach to resolve the slight tetragonality of $L 1_{0}$-ordered $\gamma$-TiAl by electron backscatter diffraction (EBSD) is presented. The phase has a $c / a$ ratio of only about $2 \%$ larger than unity. The corresponding EBSD patterns therefore exhibit cubic pseudosymmetry. As a consequence, different order variants cannot be easily distinguished on the basis of their EBSD patterns. Automated orientation mapping results in frequent misindexing. In the past, either this problem was overcome by identifying order domains by relatively laborious transmission electron microscopy, or the order domain structure was ignored altogether by using a generic face-centered cubic structure to solve for the crystal orientations, accepting a significant loss of microstructural information. The presented approach is based on the detection of the minor tetragonal distortion of the diffraction patterns by an accurate measurement of backscatter Kikuchi band positions. To this end an accurate pattern center calibration together with high-accuracy parameters for pattern acquisition and indexing are required. Together with a modified indexing algorithm, the order domains in a lamellar microstructure of $\mathrm{Ti}-45.9 \mathrm{Al}-8 \mathrm{Nb}$ (at \%) could be reliably identified. The occurrence of superlattice reflections in the Kikuchi patterns was used to validate the technique. The developed method was successfully applied to create a crystal orientation map of Ti-45.9Al-8Nb (at \%) with a fully resolved domain microstructure.
\end{abstract}

\section{Introduction}

Titanium aluminides based on the $\gamma$-TiAl phase are prospective materials for high-temperature structural components. They have a low density of around $4 \mathrm{~g} \mathrm{~cm}^{-3}$ combined with an almost constant yield strength for temperatures up to $1073 \mathrm{~K}$, and additionally good oxidation and creep resistance (Appel \& Wagner, 1998). These characteristics have led to the incipient application of $\gamma$-TiAl-based alloys in commercial aeroengines.

A good combination of properties was found for $\gamma$-TiAlbased alloys with two-phase microstructures that contain minor volume fractions of the hexagonal $\alpha_{2}-\mathrm{Ti}_{3} \mathrm{Al}$ phase. The major phase in these alloys is the $L 1_{0}$-ordered $\gamma$ phase with formula TiAl (see Fig. 1). The structure of this phase is a facecentered cubic (f.c.c.)-derived tetragonal lattice, with its (002) planes occupied by alternating layers of $\mathrm{Ti}$ and $\mathrm{Al}$, stacked along the $c$ axis of the tetragonal lattice. The $c$ axis is almost $2 \%$ longer than the $a$ axes, which leads to a $c / a$ ratio of about 1.02 , very close to unity.

Although the lattice parameters of $\gamma$-TiAl almost correspond to a cubic lattice, the orientation of the tetragonal axis of the lattice has important consequences, particularly for the deformation behavior: $\gamma$-TiAl crystals mainly deform by slip of dislocations on $\{111\}$ planes. Four dislocations with a Burgers vector of type $\frac{1}{2}\langle 110\rangle$ are called ordinary dislocations. Another eight super dislocations with Burgers vectors of type $\langle 101\rangle$ can be observed. At room temperature and in nearstoichiometric compositions, deformation by super dislocations is more difficult than that by ordinary dislocations. Additionally four twinning systems operate. All of these deformation modes have different strengths and hardening characteristics and result in pronounced plastic anisotropy of the $\gamma$ phase (Mecking et al., 1996).

\subsection{Orientation variants and domain structure of $\gamma$-TiAl}

$\gamma$-TiAl usually forms during cooling in a phase transformation from the hexagonal $\alpha$ phase (unordered) or $\alpha_{2}$ phase ( $D 0_{19}$-ordered; see e.g. Denquin \& Naka, 1996). The phase transformation occurs according to the Blackburn orientation relation of the type $(0001)_{\alpha} \|(111)_{\gamma}$ and $\langle 11 \overline{2} 0\rangle_{\alpha} \|\langle 1 \overline{1} 0\rangle_{\gamma}$, which leads to six different orientation variants as indicated in Fig. 2. These six variants fall into two groups of three. Within these groups the variants have mutually perpendicular $c$ axes. The variant triplets are called order variants. The order variants can alternatively be visualized as generated by a $120^{\circ}$ rotation around the (111) plane normal. For the notation of 
$\gamma / \gamma$ disorientations, it is sometimes useful to assume a cubic structure. This practice will be followed throughout the remainder of this work.

In a typical lamellar microstructure, created by slow cooling, each grain contains all six $\gamma$ variants. The Blackburn orientation relationship is strictly obeyed between the $\alpha_{2}$ lamellae and the $\gamma$ lamellae. The $\gamma$ phase forms a domain structure of twin-related lamellae, which themselves consist of one to three order variants. Besides the coherent twin, produced by a $180^{\circ}$ rotation around $\langle 111\rangle$, two pseudo-twin relations exist. The pseudo-twins are generated by a $\pm 60^{\circ}$ rotation around $\langle 111\rangle$, and across the pseudo-twin interface an order fault is created additionally to the change of stacking order (see Fig. 2).

To better understand not only the mechanical behavior but also the various solid state phase transformation routes of the material, characterization techniques are required which are able to reliably reveal the type and spatial arrangement of the orientational variants. Improved knowledge on the domain structure will promote the understanding of the microstructure-properties relation of titanium aluminides. The densities of different types of interfaces are the dominant variables in the application of multi-scale mechanical analyses of the Hall-Petch type (see e.g. Dimiduk et al., 1998; Maruyama et al., 2002).

\subsection{Discrimination of $\gamma$-TiAl order variants by superlattice reflections}

The ordering variants of $\gamma$-TiAl exhibit very similar diffraction patterns. Their discrimination by X-ray or electron diffraction techniques can be based on the detection of the slight tetragonal distortion of the lattice cell. Alternatively, the atomic stacking order can be analyzed by the detection of superlattice reflections. The detection of the tetragonal distortion will be discussed in the main part of this paper. The origin of superlattice reflections is briefly described in the following.

Superlattice reflections are those reflections in a diffraction pattern that are kinematically extinct in a disordered (or monoatomic) base lattice but arise when this lattice adopts an ordered structure. In the present case the disordered base lattice is an f.c.c. lattice in which the $\{110\}$ reflections, for example, are extinct owing to the kinematical extinction rules. In the tetragonal $L 1_{0}$ structure, in contrast, the $\{110\}$ reflections appear as superlattice reflections, while the $\{101\}$

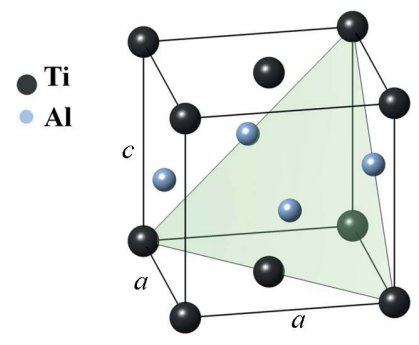

Figure 1

The crystal structure of $L 1_{0}$-ordered $\gamma$-TiAl. reflections continue to be extinct (note that in the tetragonal structure $\{110\}$ and $\{101\}$ are not equivalent).

In general, for an f.c.c. crystal of disordered structure or consisting only of a single atomic species the condition for systematic absence of diffraction intensity is that $h, k, l$ are not all odd or not all even. For the $L 1_{0}$ structure the systematic extinction is not complete in some of these cases because of the ordered arrangement of $\mathrm{Ti}$ and $\mathrm{Al}$, which possess different scattering amplitude. Under the condition that the sum of $h$ and $k$ is even and $h, k, l$ are not all even or not all odd, superlattice diffraction can be observed (Dey, Morawiec et al., 2006). From the reflectors that fulfill this condition, $\{001\}$ and $\{110\}$ have the largest intensities in electron diffraction patterns.

The determination of the domain microstructure in TiAl has for a long time been carried out by transmission electron microscopy (TEM). Here the superlattice reflections are easily recognized and can be used to determine the orientation of the $c$ axis of a given area in the microstructure. TEM selected area diffraction patterns have been used by Inui et al. (1992) to investigate the domain microstructure in lamellar Ti-49.3Al (at\%). Zaefferer (2002), for example, and Dey and co-workers (Dey, Morawiec et al., 2006; Dey, Hazotte \& Bouzy, 2006) used transmission Kikuchi diffraction patterns (TKP) to determine the crystal orientation of order domains. Unfortunately, the TEM techniques suffer from known limitations, such as small observable sample volume, complicated sample preparation and difficult practice of measurement.

An electron-diffraction-based technique that overcomes these limitations is electron backscatter diffraction (EBSD) applied in a scanning electron microscope. Instead of working in transmission on a thin foil, the technique is used to investigate the surface of bulk samples. The electron diffraction information is created in a thin layer on the surface of the material. EBSD patterns can be evaluated in the same way as TKP. However, while TKP show only a solid angle of reciprocal space of about $20^{\circ}$, EBSD patterns span under usual conditions about $70-100^{\circ}$, which allows a very wide overview of the crystal symmetry of the investigated area.

The combination of automated acquisition and analysis of EBSD patterns with scanning of the electron beam across the

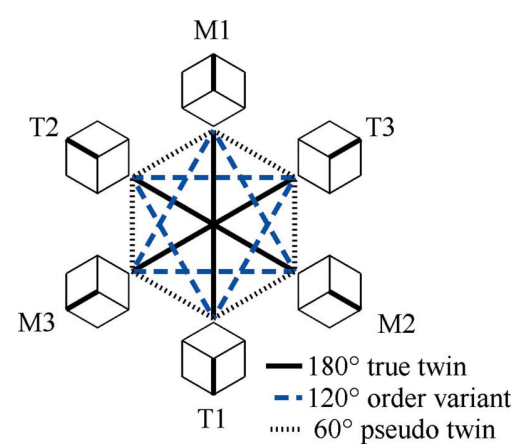

Figure 2

Orientation relationships between the six orientational variants in $\gamma$-TiAl. The $c$ axis is emphasized. Opposing variants are in a true-twin relationship, e.g. T1 and M1. The assignment of the twin (T) and matrix (M) labels is arbitrary. 
sample surface results in the orientation microscopy technique; from the orientation measured at every point of the scan grid the microstructure can be reconstructed and displayed in great detail. This very powerful microscopy tool allows the quantitative crystallographic description of large sample areas with a resolution down to approximately $50 \mathrm{~nm}$.

\subsection{Limitations of conventional EBSD analysis of $\gamma$-TiAl}

When analyzing EBSD patterns of $\gamma$-TiAl two difficulties arise. First, the large solid angle of the patterns usually precludes the observation of fine details within the patterns, for example the occurrence of superlattice reflections. As part of the automated orientation determination process, the bands within the EBSD pattern need to be detected. This is typically achieved using an image processing technique termed a Hough transform (this technique is detailed in \$2.2). The Hough transform is very efficient for determining the location of bands in the patterns; however, fine line details are veiled by the Hough transform and therefore the superlattice reflections cannot be easily detected automatically. Second, the $c / a$ ratio of $\gamma$-TiAl is so close to unity that the EBSD patterns of $\gamma$-TiAl exhibit a pseudo-cubic symmetry and the position of the $c$ axis cannot be unambiguously determined with standard algorithms. For these reasons EBSD-based orientation microscopy has, up to now, not been applied successfully to distinguish all six orientation variants of $\gamma$-TiAl microstructures.

Cubic pseudosymmetry is also observed for materials other than $\gamma$-TiAl. EBSD optimization has been performed for materials such as chalcopyrite-type semiconductors with $c / 2 a \simeq 1$ (Abou-Ras et al., 2008) and ceramic superconductors with $c / 3 a \simeq 1$ (Grossin et al., 2006).

Previous studies that applied the EBSD technique to $\gamma$-TiAl often used a cubic crystal symmetry for indexing, which can only discriminate between the twin and matrix families of variants but not between the order variants. To resolve the tetragonal orientation, these authors additionally either employed a selected area channelling pattern to identify the order variants (Simkin et al., 2003) or combined the EBSD results with a semi-automated TEM identification of the ordering domains (Dey, Morawiec et al., 2006; Dey, Hazotte \& Bouzy, 2006). Pouchou et al. (2004) increased the distance between the sample and the EBSD detector to make visible the superlattice diffraction.

Recently, Huang et al. (2007) reported that they had been able to distinguish the $c$-and $a$-axis directions, but the focus of their work lay on a massively transformed $\gamma$ phase. The massive $\gamma$ phase is known to have a c/a ratio much closer to unity than the equilibrium phase (Bartels et al., 2005). Therefore the large scatter in some of their orientation maps is not surprising. Additionally, Nave \& Inoue (2007) presented results that suggest that the tetragonal lattice can actually be resolved by EBSD. However, they did not systematically validate their results.

These previous efforts illustrate the considerable demand for a fully automatic acquisition of crystal orientation maps with accurately identified order domains. In this paper a new algorithm - based on ideas of Zaefferer (2009) - is introduced, which allows a reliable and automated determination of the correct tetragonal orientation of $\gamma$-TiAl crystals using EBSDbased orientation mapping. A careful procedure for validation of the algorithm is described. An order-domain-resolved crystal orientation map, acquired in a fully automatic manner, is presented.

\section{Enhanced accuracy EBSD pattern analysis}

The basic principle of the new approach for discrimination of TiAl order variants is the determination of the tetragonal distortion of the diffraction pattern. Three points have to be considered: first, the projection center of the pattern (often simply called the 'pattern center') needs to be known precisely, second, the positions of the Kikuchi bands need to be accurately determined and finally, an algorithm needs to be employed which determines the correct indexing for these bands.

\subsection{Pattern center calibration}

The projection center of the pattern is that point on the sample surface where the electron beam impinges, measured in detector coordinates. It is most easily calibrated using a crystal of known structure and lattice constants. In this work a silicon single crystal was used. The pattern center could then be determined by the standard procedure available in the software OIM Data Collection (OIMDC; Version 5; EDAXTSL, Draper, UT, USA). The pattern center $x, y$ and $z$ coordinates were varied, and the position for which the lowest fit value was achieved was used as the calibration setting. The fit is defined as the average angular deviation between the bands as detected by the computer and the location of the bands simulated from the measured orientation (see Nowell \& Wright, 2004).

Alternatively, the pattern center was determined using a simple optimization procedure newly implemented in the software TOCA (Zaefferer, 2002). First a sharp diffraction pattern was acquired in $O I M D C$. The positions of the Kikuchi bands were detected manually, which eliminates inaccuracies related to the Hough transformation. The bands were then indexed using an initial guess for the pattern center. Afterwards the $x, y$ and $z$ coordinates of the pattern center were systematically varied and each time the pattern was indexed and a fit value determined. The latter was calculated as the average angular deviation between the input diffraction vectors (determined from the position of the Kikuchi bands in the pattern) and the corresponding recalculated diffraction vectors after indexing and orientation determination (Zaefferer, 2002). The pattern center coordinates that result in the smallest fit value were selected and the procedure was carried out again with smaller variations, until no further decrease of the fit value occurred. This procedure did not require any assumptions about the orientation of the crystal nor did the 
first guess of the pattern center have to be good enough to obtain a correct indexing in the first step.

Both calibration methods lead to very similar results for the pattern center, usually within an error of less than $0.2 \%$ of the pattern dimensions. Fig. 3 illustrates how critical an accurate pattern center calibration is for the determination of the correct indexing of $\gamma$-TiAl variants: for this illustration the distance of the pattern center from the detector ( $z$ coordinate) was fixed to the previously determined optimum value. The figures display the dependency of the fit value on the $x y$ position of the pattern center. Each figure shows one of the three possible orientations of the $c$ axis. Solution number one corresponds to the correct orientation. This solution exhibits the lowest minimum value throughout a systematic variation of the pattern center. The other two pseudosymmetric orientations show similar behavior, with a distinct minimum at certain $x y$ positions of the pattern center. However, the observed minimum fit values are always larger than those for the correct solution. The figure illustrates the necessity of careful pattern center calibration, as the minima of the correct solution $\left(0.33^{\circ}\right)$ and the pseudosymmetric ones (0.53 and $\left.0.66^{\circ}\right)$ are very close to each other.

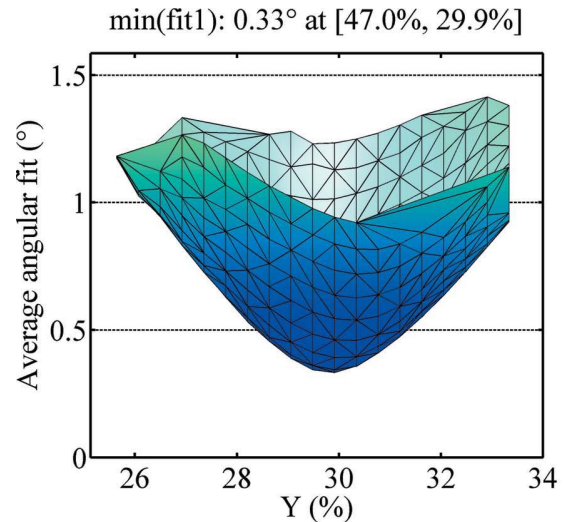

(a)

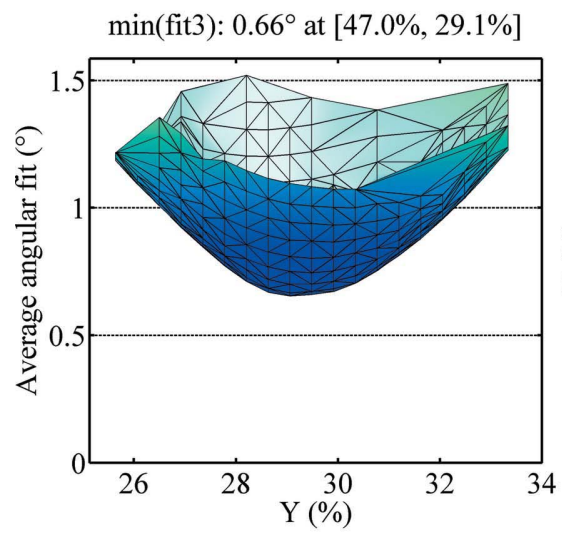

(c)

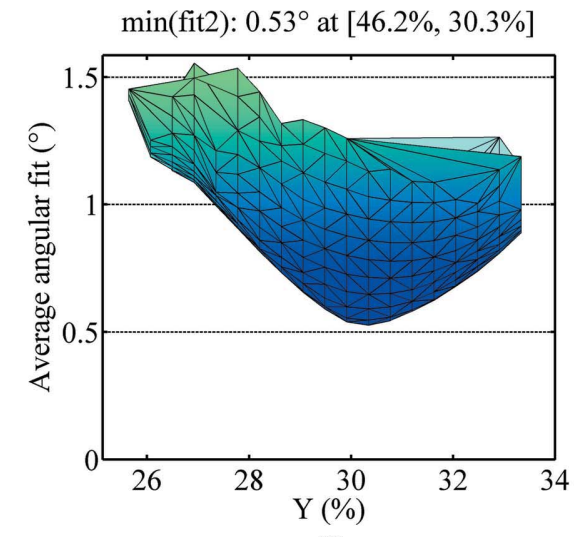

(b)

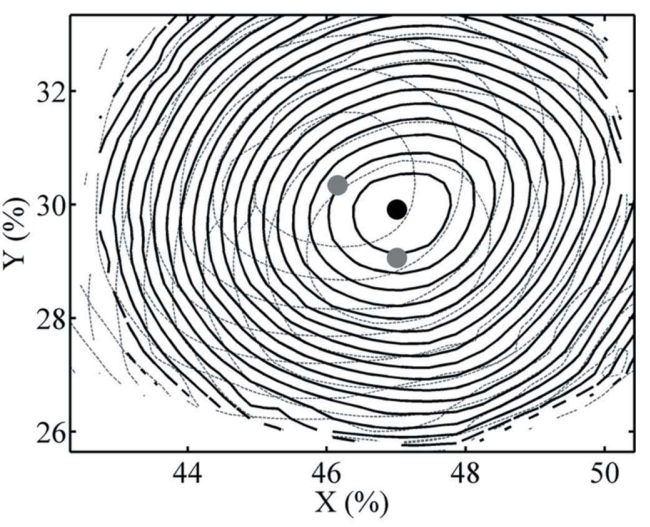

(d)
Figure 3

Sensitivity of the fit measure against variation of the pattern center coordinates in the projection plane ( $x$ and $y$ coordinates). The surface of fit values is shown for the correct indexing solution $(a)$ and the two pseudosymmetric solutions $(b)$ and $(c) .(d)$ Contour levels for the possible solutions in steps of $0.1^{\circ}$ for the pseudosymmetric solutions (gray) and $0.05^{\circ}$ for the correct solution (black). During the variation of $x$ and $y$, the $z$ coordinate of the pattern center was kept constant at its previously determined optimum position.
For the measurement of large orientation maps it is important to account for the variation of pattern center due to the scanning movement of the electron beam. This change of pattern center needs to be calibrated and corrected during the scan. For the present investigations the pattern center shift correction algorithm built into OIMDC was used.

\subsection{Accurate Kikuchi band detection}

The algorithm presented in this paper solely relies on the accurate detection of the angles between the diffraction vectors. Therefore, in order to obtain an impression of how accurate the detection has to be, it is useful to analyze the angular relations between the reflectors used for indexing. The angles between reflectors $\{h k l\}$ are listed in Table 1 . Values are given for the angular deviation between the tetragonal and the two pseudosymmetric tetragonal structures for $c / a$ ratios of 1.015 and 1.02 .

It turns out that, in order to find the correct solution from the three possible order variants, the diffraction vectors have to be determined with an accuracy of less than one degree. Within the reflectors included in Table 1 , the $\{202\}$ reflectors exhibit the maximum $c / a$ sensitivity, measured as angles between the reference and pseudo-equivalent diffraction vectors. They deviate by $1.13^{\circ}\left(0.85^{\circ}\right)$ for a $c / a$ ratio of 1.02 (1.015). The minimum nonzero sensitivity is observed for the $\{511\}$ and $\{115\}$ bands, which have an angular distance of $0.47^{\circ}$ (for $c / a=1.02$ ) and $0.36^{\circ}$ (for $c / a=$ 1.015).

The Hough transform is the standard method for identifying the Kikuchi bands in an EBSD pattern, first applied by Krieger-Lassen et al. (1992). It identifies every straight line by two parameters: the polar coordinates $\rho$ and $\theta$, where $\rho$ is the distance of the line from the center of the screen and $\theta$ is the angle of the normal vector to the band measured with respect to the horizontal axis of the screen. By summation of the intensities of all pixels along straight lines in a Kikuchi diffraction pattern and displaying this value in the parameter space $(\rho, \theta)$ (the 'Hough space') one obtains an image where each bright Kikuchi band is represented as a spot of high intensity. Since these so-called Hough peaks are created from discrete pixels in a digital image they are not sharp and well confined spots but blurred maxima of a typical shape. The detection of the position of these features occurs by convolution of the Hough image with a 


\section{Table 1}

Reflectors with a relative reflection intensity of greater than $5 \%$ of the intensity of $\{111\}$

Intensities are calculated with TOCA (Zaefferer, 2000) for Ti-50Al (at\%). Tetragonal lattice distortion is expressed by the angular deviation of pseudosymmetric diffraction vectors. The short-hand notation means that the $c$ axis of the tetragonal structure is aligned with either the $x, y$ or $z$ axis. The values for $c / a$ ratios of 1.015 and 1.02 are shown in the columns with labels 1.5 and $2.0 \%$, respectively. Superlattice reflectors are marked with an asterisk. The sensitivity of angular deviation against $c / a$ ratio is shown in the two rightmost columns; the absolute values in the four columns in the center can be calculated as sensitivity $\times[(c / a)-1]$, where $c / a$ is 1.015 or 1.020 , respectively.

\begin{tabular}{|c|c|c|c|c|c|c|c|}
\hline \multirow[b]{2}{*}{$h k l$} & \multirow[b]{2}{*}{ Intensity } & \multicolumn{2}{|c|}{$\operatorname{Angle}\left(c_{z}, c_{x}\right)\left(^{\circ}\right)$} & \multicolumn{2}{|c|}{$\operatorname{Angle}\left(c_{z}, c_{y}\right)\left(^{\circ}\right)$} & \multicolumn{2}{|c|}{$c / a$ sensitivity $\left({ }^{\circ}\right)$} \\
\hline & & $1.5 \%$ & $2.0 \%$ & $1.5 \%$ & $2.0 \%$ & $\left(c_{z}, c_{x}\right)$ & $\left(c_{z}, c_{y}\right)$ \\
\hline 111 & 1.000 & 0.69 & 0.92 & 0.69 & 0.92 & 45.71 & 45.71 \\
\hline 002 & 0.748 & 0.0 & 0.0 & 0.0 & 0.0 & 0.0 & 0.0 \\
\hline 200 & 0.715 & 0.0 & 0.0 & 0.0 & 0.0 & 0.0 & 0.0 \\
\hline 202 & 0.322 & 0.85 & 1.13 & 0.43 & 0.57 & 56.30 & 28.15 \\
\hline 220 & 0.315 & 0.43 & 0.57 & 0.43 & 0.57 & 28.15 & 28.15 \\
\hline 113 & 0.219 & 0.53 & 0.70 & 0.53 & 0.70 & 34.74 & 34.74 \\
\hline 311 & 0.211 & 0.53 & 0.70 & 0.36 & 0.48 & 34.74 & 23.67 \\
\hline 222 & 0.191 & 0.69 & 0.92 & 0.69 & 0.92 & 45.71 & 45.71 \\
\hline 004 & 0.134 & 0.0 & 0.0 & 0.0 & 0.0 & 0.0 & 0.0 \\
\hline 400 & 0.127 & 0.0 & 0.0 & 0.0 & 0.0 & 0.0 & 0.0 \\
\hline 313 & 0.102 & 0.83 & 1.10 & 0.50 & 0.67 & 54.75 & 33.13 \\
\hline $001 *$ & 0.102 & 0.0 & 0.0 & 0.0 & 0.0 & 0.0 & 0.0 \\
\hline 331 & 0.100 & 0.50 & 0.67 & 0.50 & 0.67 & 33.13 & 33.13 \\
\hline 204 & 0.097 & 0.68 & 0.91 & 0.34 & 0.46 & 45.05 & 22.76 \\
\hline 402 & 0.093 & 0.68 & 0.91 & 0.34 & 0.45 & 45.05 & 22.29 \\
\hline 420 & 0.092 & 0.34 & 0.46 & 0.34 & 0.45 & 22.76 & 22.29 \\
\hline 224 & 0.073 & 0.65 & 0.87 & 0.65 & 0.87 & 42.93 & 42.93 \\
\hline 422 & 0.071 & 0.65 & 0.87 & 0.49 & 0.65 & 42.93 & 32.13 \\
\hline $110 *$ & 0.063 & 0.43 & 0.57 & 0.43 & 0.57 & 28.15 & 28.15 \\
\hline 115 & 0.062 & 0.36 & 0.47 & 0.36 & 0.47 & 23.47 & 23.47 \\
\hline 333 & 0.060 & 0.69 & 0.92 & 0.69 & 0.92 & 45.71 & 45.71 \\
\hline 511 & 0.059 & 0.36 & 0.47 & 0.23 & 0.31 & 23.47 & 15.08 \\
\hline
\end{tabular}

particular convolution mask whose size and intensity distribution fits well to the shape of typical Hough peaks. Whenever the mask fits the intensity distribution a maximum is created in the convoluted Hough space.

The accuracy of band detection via Hough transform is determined mainly by three parameters: the resolutions of $\rho$ and $\theta$ given by $\Delta \rho$ and $\Delta \theta$, and the size of the convolution mask, $m$, where $m$ is equal to the number of rows and columns of the mask. In OIMDC the resolution of $\rho$ is determined through the number of pixels across the detector, $\rho_{\max }$. From this the angular pixel resolution is calculated as $\Delta \rho \simeq$ $2 \arctan \left(r_{\text {detector }} / z_{\text {detector }}\right) / \rho_{\text {max }}$, where $r_{\text {detector }}$ and $z_{\text {detector }}$ are the radius of the detector and the distance between the pattern center on the sample and the detector. Note that the pixel resolution changes across the screen and improves with increasing distance of a pixel from the center. The equation gives the optimal resolution value found on the rim of the detector.

The following values were used: $\rho_{\max }=240$ pixels, resulting in an angular pixel resolution of $\Delta \rho=0.375^{\circ}, \Delta \theta=0.5^{\circ}$ and $m=$ 13 pixels. The value of $m$ was selected in order to allow accurate convolution for an average Kikuchi band width at the selected detector width. A comparison of the resolution values with the required accuracy values shows that the resolution obtained with the applied Hough transform parameters is just enough to detect the tetragonal crystal orientation.
Table 2

The first five solutions for indexing a $\gamma$-TiAl backscattered Kikuchi pattern in the vote-rank algorithm as implemented in OIMDC.

By applying the fit ranking to the first three solutions, the correct solution (number three) will be assigned the first rank. The correct solution is marked with an asterisk. Orientations are given in terms of the Bunge Euler angles $\left(\varphi_{1}\right.$, $\left.\phi, \varphi_{2}\right)$.

\begin{tabular}{llrr}
\hline No. & Orientation solution $\left(\varphi_{1}, \phi, \varphi_{2}\right)\left(^{\circ}\right)$ & Votes & Fit $\left(^{\circ}\right)$ \\
\hline 1 & $(221.6,108.0,270.7)$ & 632 & 0.69 \\
2 & $(38.6,162.4,177.3)$ & 622 & 0.70 \\
$3^{*}$ & $(131.1,90.3,72.2)$ & 582 & 0.60 \\
4 & $(80.3,33.5,280.7)$ & 77 & 1.91 \\
5 & $(273.0,57.6,352.8)$ & 70 & 1.98 \\
\hline
\end{tabular}

Note here that in TiAl the $c / a$ ratio depends on the local chemical composition (Kobayashi et al., 1995; Kawabata et al., 1998) and the degree of ordering (Bartels et al., 2005). In addition, common alloying elements such as niobium show strong segregation behavior (Hecht et al., 2009). These dependencies can modify the required angular accuracy.

\subsection{Accurate and robust indexing and the fit-rank indexing method}

From the determined Hough peaks the positions of the respective diffraction vectors are calculated. Next, indexing of these diffraction vectors is performed. In OIMDC this is accomplished by selection of triplets of noncoplanar diffraction vectors. These are assigned Miller indices until their intervectorial angles match those precalculated for the crystal structure under consideration. From the indexed diffraction vector triplets the crystal orientation is easily calculated. The procedure for indexing and orientation calculation from triplets of diffraction vectors results in a number of different orientations, which are subsequently sorted and counted. The orientation with the highest number of occurrences ('votes') is selected to be the correct one (Wright \& Adams, 1992). This algorithm has been proven to be efficient and reliable for indexing EBSD patterns.

In materials with pseudosymmetry, however, this scheme results in very similar vote numbers for the pseudosymmetric orientation variants (see Table 2). Depending on the chosen set of reflectors, the indexing tolerances and the Kikuchi pattern under consideration, the standard procedure can result in an incorrect ranking of the solutions. In order to improve this behavior, a novel fit-rank method has been implemented in the OIMDC software. It is characterized by a post-processing step on the vote-rank indexing result.

First the standard vote-rank indexing as described above is applied. It identifies the three pseudosymmetric candidate orientations as those three solutions that received the highest number of votes. For each of the candidates the fit value is calculated as the angular deviation of the measured diffraction vector positions from those simulated for the determined orientation. The fit-rank method then simply consists in choosing from the three candidate solutions the one with the smallest fit value. The candidate orientation for which the 
experimentally measured band positions match the recalculated ones with highest accuracy will be selected.

\section{Experimental details}

From a sample of Ti-45.9Al-8Nb (at\%), grown in an optical floating zone furnace, a cylinder with a diameter of $5 \mathrm{~mm}$ and a height of $4 \mathrm{~mm}$ was cut by electric discharge machining. The crystal growth and cooling conditions [growth velocity $10 \mathrm{~mm} \mathrm{~h}^{-1}, \quad 10^{5} \mathrm{~Pa}$ argon atmosphere; for further details see Souptel et al. (2007)] led to the formation of a columnar microstructure with lamellar grains of about $500 \mu \mathrm{m}$ grain size. After grinding to 1000 grit and a successive polishing with $3 \mu \mathrm{m}$ diamond paste, electrolytic polishing was carried out to remove any remaining surface deformation. A solution of $6 \%$ perchloric acid in ethanol was used as electrolyte. Polishing was carried out with a voltage of $35 \mathrm{~V}$ at a temperature of $243 \mathrm{~K}$.

A Schottky-type field-emission gun scanning electron microscope (JEOL JSM $6500 \mathrm{~F}$ ) was used for acquisition of EBSD data. The scanning electron microscope was operated at an acceleration voltage of $30 \mathrm{kV}$ and the sample was tilted to $70^{\circ}$ out of the horizontal position. The working distance was $15 \mathrm{~mm}$.

An EDAX/TSL EBSD acquisition system with a DigiView II camera was used for pattern recording. The recording phosphor screen had a diameter of $40 \mathrm{~mm}$ and was positioned at a distance of $19.1 \mathrm{~mm}$ from the pattern source. Diffraction patterns were recorded at a resolution of $936 \times 936$ pixels and an exposure time of $0.5 \mathrm{~s}$. For acquisition and processing of the patterns the commercial software system OIMDC was employed. For accurate line detection a high-resolution Hough transform of 240 pixels $\times 0.5^{\circ}$ (resolution in $\theta$ and $\rho$ ) and a large convolution mask $(13 \times 13$ pixels $)$ were applied. Sixteen bands were used for the subsequent indexing. The interplanar angle tolerance of the vote indexing was set to a value of $3^{\circ}$. The following reflectors were included for indexing the patterns: $\{001\},\{111\},\{200\},\{202\},\{220\},\{113\}$, $\{311\},\{313\},\{331\},\{024\},\{402\},\{240\}$.

\section{Results and discussion}

\subsection{Validation of the proposed fit-rank method}

Before applying the fit-rank method the pattern center was accurately determined as described in \$2.1. Having determined the correct pattern center the three possible solutions can be differentiated by their fit value. Table 2 shows the number of votes as well as the average angular fit value for the first five solutions obtained by a representative indexing run in OIMDC. Clearly, the first three orientations have a significantly higher number of votes when compared with the following ones and are therefore the three candidate orientations. The proposed fit-rank procedure correctly promotes solution number three, which has the lowest number of votes, to the first position.

In order to validate the approach, it is necessary to have an independent check for the correct orientation. A separate

Figure 5

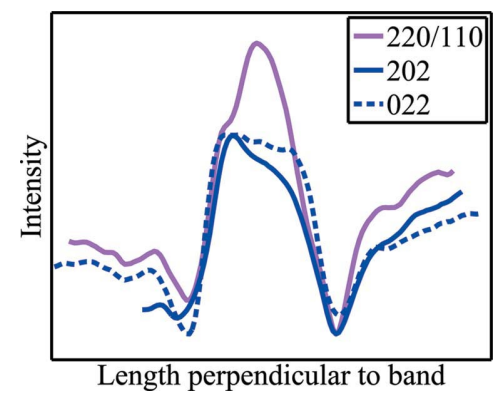

Intensity profiles of the $220 / 110$ reflections and the 202 and 022 reflections in the pattern shown in Fig. 4(b). 
Table 3

The first five indexing solutions for the two patterns shown in Fig. 4.

Each pattern was calibrated individually. 12 Hough peaks were taken into account. Interplanar angle tolerance $3^{\circ}$.

\begin{tabular}{lrrrrrr}
\hline & \multicolumn{2}{l}{ Pattern from Fig. $4(b)$} & & \multicolumn{2}{l}{ Pattern from Fig. 4(c) } \\
\cline { 2 - 3 } Solution & \multicolumn{1}{l}{ Votes } & Fit $\left({ }^{\circ}\right)$ & & \multicolumn{2}{l}{ Votes } & Fit $\left({ }^{\circ}\right)$ \\
\hline 1 & 220 & 0.26 & & 220 & 0.25 \\
2 & 210 & 0.54 & & 200 & 0.65 \\
3 & 182 & 0.51 & & 189 & 0.65 \\
4 & 14 & 1.92 & & 12 & 1.90 \\
5 & 14 & 2.02 & & 12 & 2.01 \\
\hline
\end{tabular}

method of identifying the order variant is given by the observation of the superlattice reflections. Exposure times of the order of one second were used to collect the EBSD patterns. At these exposure times $\{110\}$ superlattice reflections start to become visible inside $\{220\}$ bands. The superlattice bands, being half as wide as the $\{220\}$ bands, appear as faint internal structure of higher intensity. An example is given in Fig. 4. Here two EBSD patterns from two neighboring lamellae with different order variants are shown. In both patterns the positions of the $\{220\}$ and $\{202\}$ base reflections are marked by arrows. The $\{220\}$ reflection which contains the $\{110\}$ superlattice reflection is marked by a magenta arrow. The superlattice band could be recognized although the calculated kinematical intensity (Table 1) of the superlattice band is only about $20 \%$ of the intensity of the $\{220\}$ bands in stoichiometric TiAl. In the present case, where 8 at $\% \mathrm{Nb}$ replaces $\mathrm{Ti}^{\mathbf{1}}{ }^{1}$ the intensities of the superlattice reflections are actually higher. With increasing $\mathrm{Nb}$ content, the intensity of the superlattice reflection increases approximately proportionally to the $\mathrm{Nb}$ content. In the case where all of the $\mathrm{Ti}$ is replaced by $\mathrm{Nb}$, the theoretical intensity of the $\{110\}$ bands is simulated to be $60 \%$ of the intensity of the $\{220\}$ bands.

Since the existence of the superlattice reflection may not be easily visible to the reader, Fig. 5 displays the integrated intensity profile of the (220)/(110) reflection and the (202) and (022) reflections along a part of their extension in Fig. 4(b). Although the structure of the superlattice reflection is not fully resolved, a distinct additional intensity contribution from (110) is obvious for the position inside the (220) reflection.

On the right-hand side of Fig. 4 the three respective indexing solutions are shown overlaid on the diffraction pattern. The determined numbers of votes as well as the angular fit values are given in Table 3 . The first-ranked solutions with the highest number of votes and simultaneously the lowest fit value are the solutions with the correct position of the superlattice reflection. In this case the vote-rank indexing and the fit-rank indexing yield identical results.

For a more systematic validation, a line scan was carried out. It consisted of 400 patterns taken with a step size of $0.2 \mu \mathrm{m}$ along a horizontal line through the center of the microstructure discussed in $\$ 4.2$. The patterns were collected with an exposure time of $1.2 \mathrm{~s}$ to allow visual identification of

${ }^{\mathbf{1}}$ Niobium preferentially occupies Ti sites in the equilibrium phase (see Scheu et al., 2007, and references therein). the $\{110\}$ superlattice reflections. Fig. 6 shows the results of the validation procedure. From $80 \%$ of the recorded patterns the position of the $\{110\}$ superlattice bands could be identified with confidence by visual inspection. The remaining $20 \%$ were

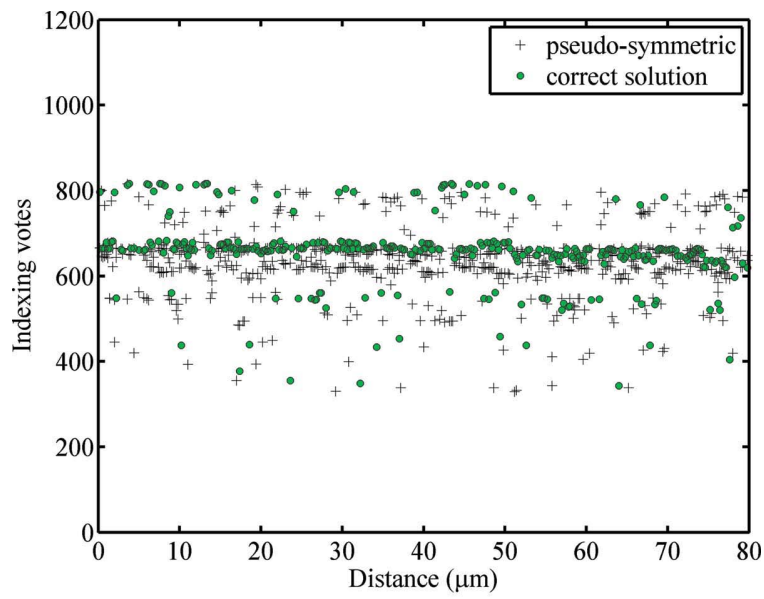

(a)

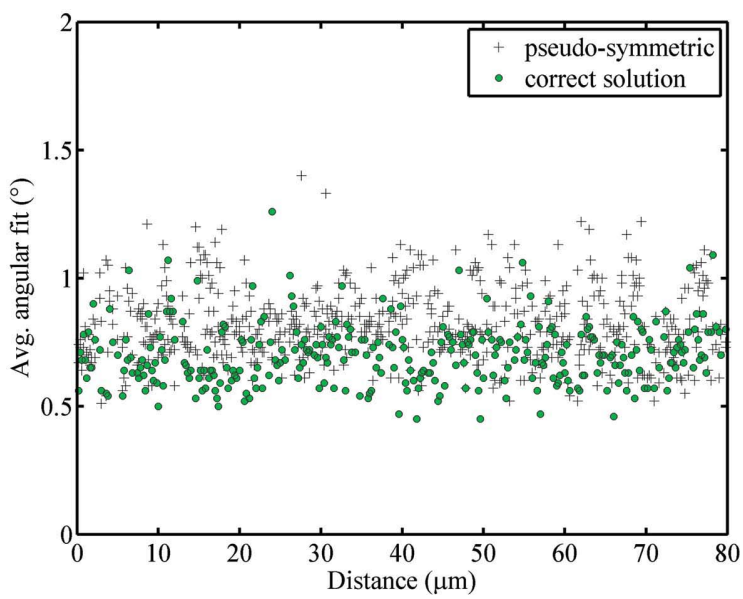

(b)

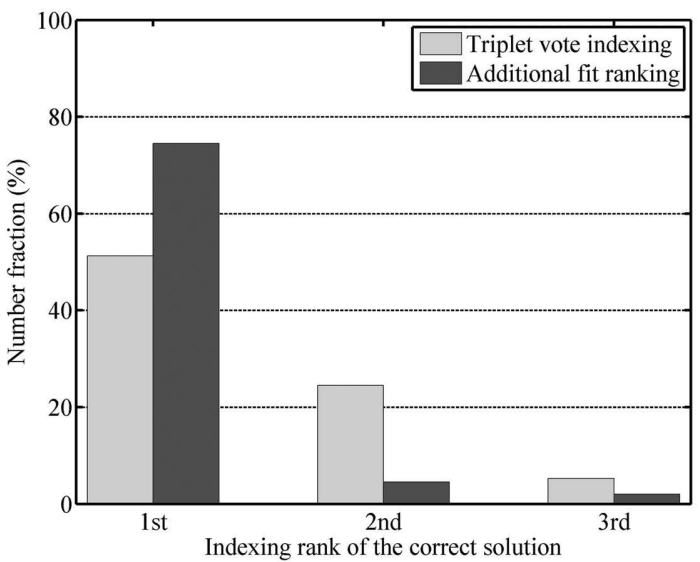

(c)

Figure 6

(a) Indexing votes and (b) angular fit values for the recorded line data. The filled circles mark the correct solution; gray plus signs display the indexing quality measures for the pseudosymmetric orientations. (c) The overall performance of the fit-rank method versus the pure vote indexing, applied to the validation data set. 


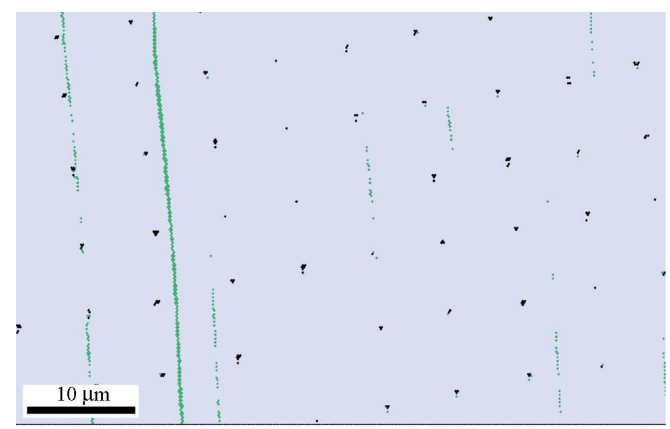

(a)

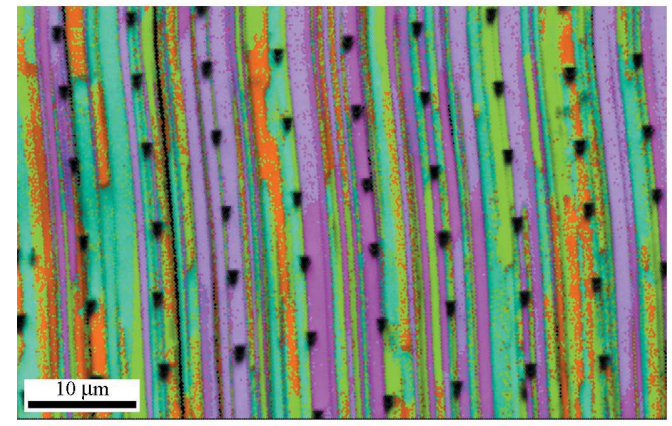

(c)

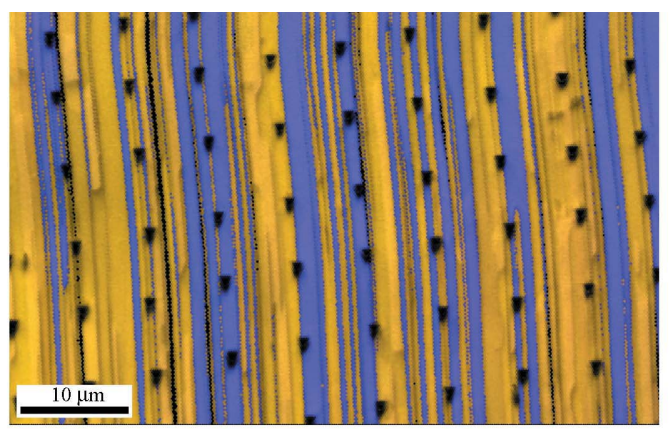

(b)

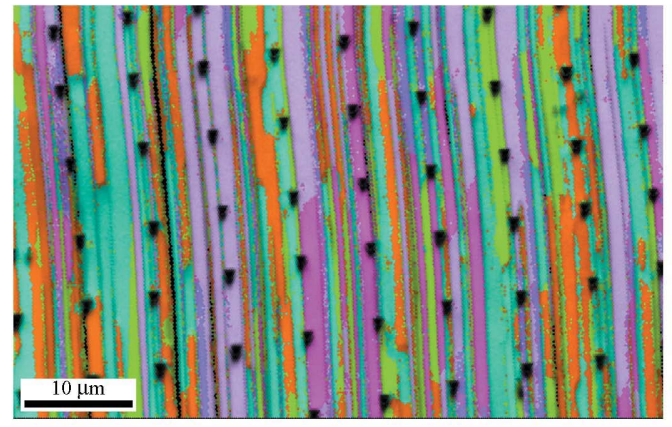

$(d)$
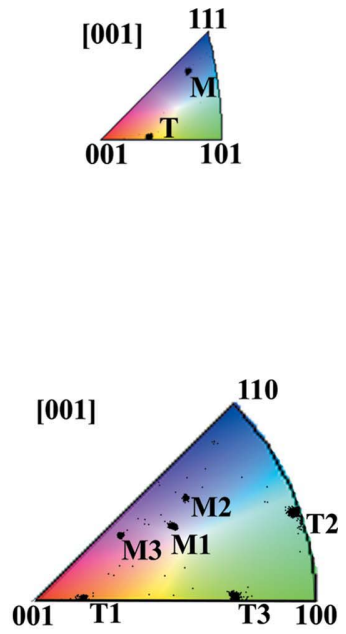

Figure 7

EBSD maps of lamellar Ti-45.9Al-8Nb (at \%), measured with step size of $0.2 \mu \mathrm{m}$ (hexagonal grid): (a) phase map, light gray is $\gamma$-TiAl, green is hexagonal $\alpha_{2}-\mathrm{Ti}_{3} \mathrm{Al} ;(b)$ indexing with f.c.c. structure, inverse pole figure (IPF) coloring scheme (see legend) combined with a grayscale image of the band contrast $(\mathrm{BC}) ;(c)$ vote-rank indexing result, IPF\&BC colors; $(d)$ fit-rank indexing result, IPF \&BC colors. The black markings, indicating very low band contrast, are nanoindentations which made it possible to locate positions precisely in the microstructure during an interactive scanning electron microscope observation.

obtained from areas close to $\gamma / \gamma$ or $\alpha_{2} / \gamma$ boundaries. These patterns showed overlapped patterns or poor contrast and the $\{110\}$ band position could not be determined by eye.

Fig. 6(a) illustrates the number of votes that were given to the correct solution as identified manually. The vote numbers given to the pseudosymmetric solutions are also shown. Ideally, the correct solution would receive the highest number of votes for each measurement. However, during automatic indexing, the conventional vote-rank indexing identified only $50 \%$ of the 400 patterns correctly. The distribution of fit values between the correct and the pseudosymmetric solutions is displayed in Fig. $6(b)$. When the newly developed fit-based

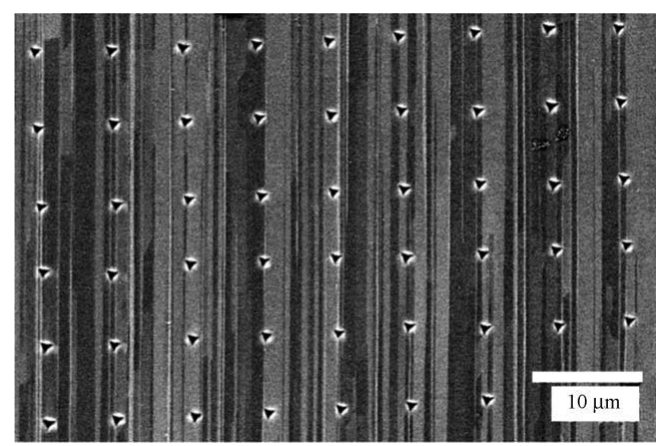

Figure 8

Backscattered electron image of the microstructure shown in the orientation maps. $\alpha_{2}-\mathrm{Ti}_{3} \mathrm{Al}$ appears bright, while $\gamma$-TiAl produces different shades of gray. solution ranking was applied, the ratio of successfully identified orientations increased to about $75 \%$. Keeping in mind that only $80 \%$ of all patterns could be identified correctly by the manual procedure this is an excellent rate of correct indexing. Fig. 6(c) compares the fraction of correctly indexed points and the fractions of misindexed patterns for the two methods.

Taking as a reference only those $80 \%$ of the 400 patterns that could be unambiguously indexed in the manual procedure, the fraction of correctly indexed patterns rises to $94 \%$. This high ratio of correct indexing represents the upper limit in the case of excellent pattern quality. In conclusion, for an automatic mapping with applied fit-rank procedure, the fraction of correctly indexed patterns is expected to be in the range of $75-94 \%$ in comparison with an upper bound of $63 \%$ for pure vote indexing.

\subsection{Orientation mapping of the order domain structure}

The developed fit-rank method has been applied to a lamellar microstructure of Ti-45.9Al-8Nb (at\%). Fig. 7 displays the results, measured at a step size of $0.2 \mu \mathrm{m}$ on a hexagonal grid. The phase distribution map shown in Fig. 7(a) illustrates the prevalence of the $\gamma$ phase in contrast to the thin lamellae consisting of $\alpha_{2}-\mathrm{Ti}_{3} \mathrm{Al}$. Of the measured points, $2 \%$ are assigned to the $\alpha_{2}$ phase. This $\alpha_{2}$ phase volume fraction is underestimated because of the limited resolution of the EBSD technique at high acceleration voltage. On the thin $\alpha_{2}$ 
lamellae, patterns from the surrounding $\gamma$ phase frequently overlap with the $\alpha_{2}$ pattern, thus leading to identification of the $\gamma$ phase. The minor curvature of the lamellar structure is an artefact due to specimen drift during the relatively long measurement time of about nine hours. The backscattered electron image in Fig. 8 shows some thin $\alpha_{2}$ lamellae not visible in Fig. 7(a), as well as perfectly flat lamellar interfaces.

Fig. 7(b) shows an orientation map for indexing with a generic f.c.c. structure. Only the two twin-related variant families can be distinguished. One family of order domains comprises $36 \%$ of the mapped points, here arbitrarily denoted by ' $\mathrm{M}$ ' for matrix. The twin-related family (' $T$ '), shown in ochre, has an area fraction of $62 \%$. The order variants of twin or matrix type tend to occur grouped together. In the orientation map Fig. 7(b) this aggregation is more pronounced for the twin-type variants than for the matrix-type orientations. The clustered appearance of order variants has previously been termed 'one twin dominant zones' (Dey, Hazotte \& Bouzy, 2006).

Figs. 7(c) and 7(d) show the orientation maps as obtained with a tetragonal structure $(c / a=1.02)$ by using the vote-rank and the fit-rank indexing algorithm, respectively. The order domains are clearly resolved in both cases. However, Fig. 7(c) shows a higher proportion of isolated points of differing orientation. These are due to the selection of the wrong order variant by the vote-rank method. Fig. $7(d)$, which is calculated using the fit-rank method, gives a clear picture of the order domain structure. No post-processing step to clean up the data was applied. Very few isolated points - where the wrong order variant has been selected by the indexing software - remain in this map obtained with the fit-rank method.

A good indication of the correct detection of the order domain structure is given by the distribution of pattern quality. The position of order domain boundaries is indicated by a reduced sharpness of the Kikuchi bands which results from misfit stresses and defects along the boundaries and therefore lower values in the pattern quality maps. In Fig. $7(d)$ the identified domain boundaries follow the lines of lower band contrast. They appear darker because the band contrast is overlaid in the form of gray levels.

In addition, the order domain structure was compared with the backscattered electron image in Fig. 8. It shows the same region as the EBSD results. The $\alpha_{2}$ phase appears bright. Different orientations of $\gamma$-TiAl can be discriminated by their gray-level contrast. A comparison shows very good agreement with the EBSD orientation maps.

Twin boundaries are observed to be almost exclusively aligned with the lamellae, while order domains may change along the lamellae. The width of the order domains is about 1$2 \mu \mathrm{m}$. The lamellae are inclined to the surface at an angle of $68^{\circ}$. In Fig. $7(d)$ the length of order domain boundaries [120 (1) ${ }^{\circ}$ rotation for a $\langle 111\rangle$ plane normal] was analyzed to be $3.52 \mathrm{~mm}$. Converted to boundary length per area $(60 \times 38 \mu \mathrm{m})$, this value corresponds to $1.55 \mu^{-1}$.

It is emphasized here again that an accurate pattern center calibration is essential for obtaining correct data. For an incorrect calibration the fit rank indexing may produce a microstructure that looks correct but which might nevertheless contain a significant number of wrongly indexed points. Therefore, the conditions of the setup have to be checked before each scan by manual evaluation of individual backscattered Kikuchi patterns. This on-site validation should be performed with patterns taken at longer exposure times, which exhibit the mentioned $\{110\}$ superlattice reflection.

\subsection{Further improvement of the algorithm}

It is possible that the use of appropriate, particularly sensitive reflectors (see Table 1) could enhance the proposed indexing scheme. This could, for example, be achieved by assigning a higher weight to these sensitive reflectors during the calculation of the average angular fit measure.

Certainly the selection of higher Hough transform resolution is another possibility for further development. A fundamental problem with the current Hough transform approach is caused by the use of a static convolution mask. First, as a result of its constant size an accurate band position detection is only possible for a limited range of Kikuchi band widths. Furthermore, the use of a convolution mask veils the contrasts of superlattice reflections, which could otherwise be used to check the correctness of the solution. A possible way around these problems is the use of the Hough backmapping transformation as introduced by Krieger-Lassen (1998). The Hough backmapping transformation results in a very sparsely filled Hough space so that the band or line maxima can be detected without further filtering of the image.

It might be possible to adjust the internal tolerances of the vote-rank procedure to identify the order domains. For example, a decrease of the interplanar angle tolerance parameter, as defined in the software $O I M D C$, was observed to reduce the number of misindexed points. However, in the current setting, when the calibration of the EBSD system is carried out with high accuracy, the average angular fit measure was found to be more sensitive to the correct solution than the number of votes.

In general, the proposed two-step procedure seems to be computationally most efficient for situations in which crystal orientations related to very similar Kikuchi patterns need to be discriminated. First, the robust and fast vote-rank method is applied to find a finite number of candidate solutions. Afterwards, a tailored criterion such as the fit-rank or possibly a computationally more demanding evaluation of the candidates can be applied.

\section{Conclusions}

A new approach to resolve the slight tetragonality of $L 1_{0^{-}}$ ordered $\gamma$-TiAl by electron backscatter diffraction was presented. The new algorithm enables the reliable identification of order domains in $\gamma$-TiAl-based alloys.

The approach is based on the detection of the tetragonal distortion of the diffraction patterns. An accurate pattern center calibration together with high-accuracy parameters for pattern acquisition and indexing were employed. The applied 
Hough transform parameters were just sufficient to resolve the slight tetragonal distortion of the material, but a more highly resolved Hough transform would be beneficial for still more reliable data analysis.

The average angular fit between the measured and simulated band positions was shown to be a more sensitive parameter for the identification of TiAl order domains than the triplet vote measure. The approach was successfully validated and indexing of a crystal orientation map was performed with a newly developed fit-rank algorithm. It was shown that, with the developed method, the rate of successful indexing in $\gamma$-TiAl can reach values of up to around $90 \%$ in a fully automatic orientation mapping.

Dr G. Behr and Dr W. Löser (IFW Dresden) are acknowledged for providing the sample material. D. Steinmetz (MPIE) is thanked for his help on computer automation. Support by the European Union FP6 integrated project 'Intermetallic Materials Processing in Relation to Earth and Space Solidification' (IMPRESS) under contract NMP3-CT-2004-500635 is highly appreciated.

\section{References}

Abou-Ras, D., Gibmeier, J., Nolze, G., Gholinia, A. \& Konijnenberg, P. (2008). Cryst. Res. Technol. 43, 234-239.

Appel, F. \& Wagner, R. (1998). Mater. Sci. Eng. R, 22, 187-268.

Bartels, A., Bystrzanowski, S., Chladil, H., Leitner, H., Clemens, H., Gerling, R. \& Schimansky, F.-P. (2005). Mater. Res. Soc. Symp. Proc. 842, S5.48.

Denquin, A. \& Naka, S. (1996). Acta Mater. 44, 343-352.

Dey, S. R., Hazotte, A. \& Bouzy, E. (2006). Philos. Mag. 86, 30893112.
Dey, S. R., Morawiec, A., Bouzy, E., Hazotte, A. \& Fundenberger, J. J. (2006). Mater. Lett. 60, 646-650.

Dimiduk, D. M., Hazzledine, P. M., Parthasarathy, T. A., Seshagiri, S. \& Mendiratta, M. G. (1998). Metall. Mater. Trans. A, 29, 37-47.

Grossin, D., Henrist, C., Mathieu, J.-P., Meslin, S., Harnois, C., Noudem, J.-G., Cloots, R. \& Chateigner, D. (2006). Supercond. Sci. Technol. 19, 190-199.

Hecht, U., Daloz, D., Lapin, J., Drevermann, A., Witusiewicz, V. T. \& Zollinger, J. (2009). Mater. Res. Soc. Symp. Proc. 1128, U03-01.

Huang, A., Hu, D., Wu, X. \& Loretto, M. (2007). Intermetallics, 15, 1147-1155.

Inui, H., Oh, M. H., Nakamura, A. \& Yamaguchi, M. (1992). Philos. Mag. A, 66, 539-555.

Kawabata, T. Fukai, H. \& Izumi, O. (1998). Acta Mater. 46, 21852194.

Kobayashi, E., Shinmoto, K., Miura, S., Suzuki, T. \& Mishima, Y. (1995). Gamma Titanium Aluminides 1995, pp. 347-352. Warrendale: TMS.

Krieger-Lassen, N. C. (1998). J. Microsc. 190, 375-391.

Krieger-Lassen, N. C., Juul-Jensen, D. \& Conradsen, K. (1992). Scanning Microsc. 6, 115-121.

Maruyama, K., Suzuki, G., Kim, H. Y., Suzuki, M. \& Sato, H. (2002). Mater. Sci. Eng. A, 329, 190-195.

Mecking, H., Hartig, C. \& Kocks, U. F. (1996). Acta Mater. 44, 13091321.

Nave, M. \& Inoue, H. (2007). Mater. Sci. Forum, 561-565, 431-434.

Nowell, M. M. \& Wright, S. I. (2004). J. Microsc. 213, 296-305.

Pouchou, J.-L., Denquin, A. \& Ferrini, A. (2004). Microchim. Acta, 145, 177-182.

Scheu, C., Cha, L. M., Sturm, S., Chladil, H. F., Mayrhofer, P. H., Clemens, H., Wole, W. \& Podloucky, R. (2007). Mater. Res. Soc. Proc. 980, 401-406.

Simkin, B. A., Ng, B. C., Bieler, T. R., Crimp, M. A. \& Mason, D. E. (2003). Intermetallics, 11, 215-223.

Souptel, D., Löser, W. \& Behr, G. (2007). J. Cryst. Growth, 300, 538550 .

Wright, S. I. \& Adams, B. L. (1992). Metall. Trans. A, 23, 759-767.

Zaefferer, S. (2000). J. Appl. Cryst. 33, 10-25.

Zaefferer, S. (2002). Adv. Imaging Electron Phys. 125, 355-415.

Zaefferer, S. (2009). Habilitation thesis, RWTH Aachen, Germany. 